\title{
Resolvability for Imprecise Multi-attribute Alternative Selection
}

\author{
Chelsea C. White III, Fellow, IEEE \\ Hillary A. Holloway
}

\begin{abstract}
We consider an alternative selection process that involves multiple and conflicting objectives. A facilitator asks a decision-maker (DM), or a decision-making body, questions and obtains responses in order to help the DM select a most preferred alternative. The DM responses to these questions do not necessarily result in precisely determined parameter values (e.g., weights and value scores corresponding to a given model of preference). The goal of this research is to guide the facilitator in selecting what question to ask next, and determining when to terminate the question-response process.

We model the facilitator's question selection problem as a Markov decision process, and present conditions that guarantee the existence of a question-response policy to identify a most preferred alternative in a finite number of questions. We also present conditions that guarantee the existence of a questionresponse policy that will identify a most preferred alternative as the number of questions asked goes to infinity. Both of these sets of conditions are easily identified a priori and may be used to aid a facilitator in determining which questions to ask, and when to terminate the alternative selection process because identification of a most preferred alternative is not possible.
\end{abstract}

Index Terms-Decision-aiding, ISMAUT, question selection.

\section{INTRODUCTION}

The research reported in this paper was motivated by an unanticipated situation that first emerged during a real-world group alternative selection session. Unknown to either the facilitator or the members of the group at the time, it was impossible for a most preferred alternative to be identified for the alternative selection problem under consideration. In retrospect, this inability to identify a most preferred alternative was due to a restriction during the session to assess only tradeoff weights and to the imprecision of some of the value scores, all of which were previously assessed by individuals not in the group. The group was intent on, and expected to, determine a most preferred alternative. When a most preferred alternative was not found after a considerable investment of time and effort in determining precise trade-off weights, the session was perceived a failure.

This experience raised several questions: Assuming parameter imprecision is permissible, what are the conditions under which there exists a question-response policy (i.e., a procedure that determines what question to ask next) that will lead to a most preferred alternative? If these conditions do not hold,

Chelsea C. White III is H. Milton \& Carolyn J. Stewart School Chair and Schneider National Chair of Transportation and Logistics at the Georgia Institute of Technology. E-mail: cwhite@isye.gatech.edu.

Hillary A. Holloway is Lead Engineer at BAE Systems Advanced Information Technologies in Burlington, Massachusetts. E-mail: holloway@post.harvard.edu. what is the probability that there is no question-response policy that will lead to a most preferred alternative? In either case, and assuming we continue to seek a most preferred alternative, what policy should we use? The answers to these questions could help a facilitator better manage a group's time, effort, and expectations and better determine when to stop a session. In addressing these questions, the research in this paper provides a priori conditions that guarantee that a most preferred alternative can be found, and for situations where this is not the case, provides techniques for determining the probability that a most preferred alternative cannot be identified. Dynamic programming is used for policy determination.

In the subsection that follows, we describe the alternative selection process in more detail, and provide both a simple example that illustrates the aforementioned situation, and a more specific description of the group alternative selection session in which the situation emerged. We then present a literature review and outline the remainder of the paper.

\section{A. A model of decision-making and the Question Selection Problem}

For the alternative selection process under consideration there is a decision-maker (DM), or a decision-making body, and a facilitator. The facilitator's goal is to help the DM select a most preferred alternative from a finite set of alternatives when multiple and conflicting objectives may be under consideration. In order to achieve this goal, the facilitator gathers information from the DM by asking the DM questions and obtaining responses. For group decision-making, we assume throughout that all members of the group agree with the response. The facilitator must decide what question to ask next, which we refer to as the Question Selection Problem (QSP).

Furthermore, the facilitator may need to decide when to terminate the QSP. This decision is trivial once a most preferred alternative has been identified, or equivalently, once the QSP has been resolved. However, there are situations, illustrated later, where the question-response process is not able to further the search for a most preferred alternative. Once such a situation is identified, then it behooves the facilitator to terminate the process in order to save the DM unnecessary time and effort. Such situations are said to leave the QSP unresolved.

The underlying model of alternative selection that serves as a basis for our research is an extension of Multi-Attribute Utility Theory (MAUT; Keeney \& Raiffa [1]), called Imprecisely 
Specified MAUT (ISMAUT)[2], [3]. In this paper, we consider the special case where each alternative results in a single consequence, and as a result, we refer to a utility function as a value function. The simplest MAUT model under this assumption involves:

- a finite number of alternatives, $A$;

- a finite number of attributes, $I$;

- a trade-off weight for each attribute, $w_{i}$ for attribute $i \in I$;

- a value score for each attribute and alternative, $v_{i}(a)$ for alternative $a \in A$ and attribute $i \in I$;

- the total value of alternative $a \in A$ is given by the weighted sum $w v(a)=\sum_{i} w_{i} v_{i}(a)$.

Further we assume:

- for all $i \in I, w_{i} \geq 0$, and $\sum_{i} w_{i}=1$;

- for all $a \in A$ and $i \in I, v_{i}(a) \in[0,1]$, and for each $i \in I$ there are $a^{\prime}, a^{\prime \prime} \in A$ such that $v_{i}\left(a^{\prime}\right)=1$ and $v_{i}\left(a^{\prime \prime}\right)=0$;

- alternative $a^{\prime}$ is at least as preferred as alternative $a^{\prime \prime}$ if and only if $w v\left(a^{\prime}\right) \geq w v\left(a^{\prime \prime}\right)$.

The problem objective is to find an alternative $a^{*} \in A$ such

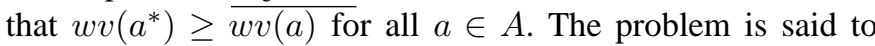
be resolved once the problem objective is achieved.

Use of MAUT for this simple model typically involves the following facilitation process:

- Identify $A$ and $I$.

- For each $i$, identify a most preferred alternative $a^{\prime}$ and a least preferred alternative $a^{\prime \prime}$, and then determine the $v_{i}(a)$ for all $a \in A$.

- Identify the trade-off weights.

- Use $w v(a), a \in A$, to (totally) order the alternatives.

There are three observations that are appropriate to make at this point. First, the additive value function that is used assumes preferential independence between the attributes (see [1] for definitions and further discussion). Second, the structure of the above facilitation process is relatively straightforward. Third, difficulty may occur in eliciting precise numbers for the value scores and trade-off weights from the DM. As Keeney and Raiffa [1] point out, achieving the precision in values necessary for identification of a total weighted value/utility maximizing decision requires asking questions that DMs are not always comfortable answering. In particular, DMs are often reluctant to gauge their preferences with precise numbers. For example, a hypothetical dialogue from their book [1, p. 98] involves the DM answering a question with the words, "I don't know. I would say about 60 . But I feel awfully woozy about that." The authors point out the discomfort they have encountered when human DMs have been forced to commit to a particular number to describe their preferences. (See Bard [4] for a case study and additional illustration of difficulties encountered using MAUT.)

It has been our experience that DMs are often more comfortable in making natural language statements during the elicitation process that can be interpreted as linear inequalities on the trade-off weights and value scores. Examples of such statements are:

- "The Cadillac is better looking than the Pinto."

- "Safety is more important than cost."
This third observation motivated the development of ISMAUT, which in its simplest form allows for trade-off weights and value scores to be described by finite sets of linear inequalities, or more generally by set inclusion as follows. Assume that all we know about $w=\left\{w_{i}, i \in I\right\}$ and $v_{i}=\left\{v_{i}(a), a \in A\right\}, i \in I$, is that $w$ is an element of some set $W, v=\left\{v_{i}\right\}$ is an element of some set $V=\left\{V_{i}\right\}$, and $W$ and $V_{i}$ are given for all $i$. Then we say alternative $a^{\prime}$ is at least as preferred as alternative $a^{\prime \prime}$ if and only if $w v\left(a^{\prime}\right) \geq w v\left(a^{\prime \prime}\right)$ for all $w \in W$ and $v \in V$. This (very conservative and partial) ordering on the alternatives leads to situations where (1) there may be several alternatives that are candidates for being most preferred and hence where (2) the DM wishes to continue the alternative selection process until this set of candidate alternatives, i.e. the non-dominated set, is reduced further, ideally to problem resolution.

It is straightforward to show that if $W^{\prime} \subseteq W$ and $V^{\prime} \subseteq$ $V$, then the non-dominated set of alternatives generated by $\left(V^{\prime}, W^{\prime}\right)$ will be a (not necessarily strict) subset of the nondominated set generated by $(V, W)$. Thus it is usually useful to ask the questions regarding DM preferences in order to obtain a more precise understanding of the value scores and tradeoff weights. This discussion suggests the sequential process depicted in the flow chart shown in Figure 1, where we let $S=(V, W)$ and denote the non-dominated set by $N D(S)$.

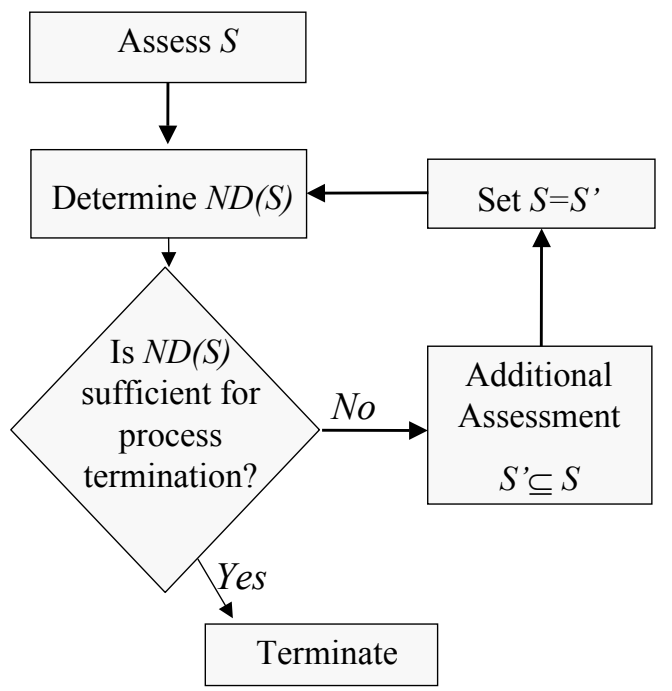

Fig. 1. Flowchart of the decision process

We remark that reducing value score and trade-off weight imprecision can be time consuming and stressful, and can generate conflict within a group. Thus, there may be value in limiting the process of imprecision reduction and in identifying when no such reduction is useful. With this in mind, we remark that the facilitation process associated with ISMAUT can be considerably more complex than that associated with MAUT, and hence there may be need to support the ISMAUT facilitator. We illustrate these issues with the following example.

EXAMPLE 1. Assume there are three alternatives, $A=$ 
$\left\{a_{1}, a_{2}, a_{3}\right\}$, and two attributes, $I=\left\{i_{1}, i_{2}\right\}$, under consideration. Further, assume the elicitation process has thus far produced the matrix of value scores shown in Table I, where the value score $v_{1}\left(a_{2}\right)$ has yet to be elicited.

\begin{tabular}{|c|c|c|}
\hline Alternatives & $i_{1}$ & $i_{2}$ \\
\hline$a_{1}$ & 1 & 0 \\
$a_{2}$ & $v_{1}\left(a_{2}\right)$ & 0.3 \\
$a_{3}$ & 0 & 1 \\
\hline
\end{tabular}

TABLE I

VALUE SCORES FOR THREE ALTERNATIVES

The standard MAUT procedure would elicit $v_{1}\left(a_{2}\right)$ and then elicit the trade-off weight $w_{1}$. Because $w_{2}=1-w_{1}$, this would be sufficient to determine a total preference ordering of the alternatives. ISMAUT can consider several additional ways in which the alternative selection process could proceed. For example, let $S_{a}=\left\{(v, w): w v(a) \geq w v\left(a^{\prime}\right) \forall a^{\prime} \in A\right\}$. Thus, $(v, w) \in S_{a}$ if and only if the alternative $a$ is a most preferred alternative. A graphical depiction of $S_{a}$, for all $a \in A$, is presented in Figure 2.

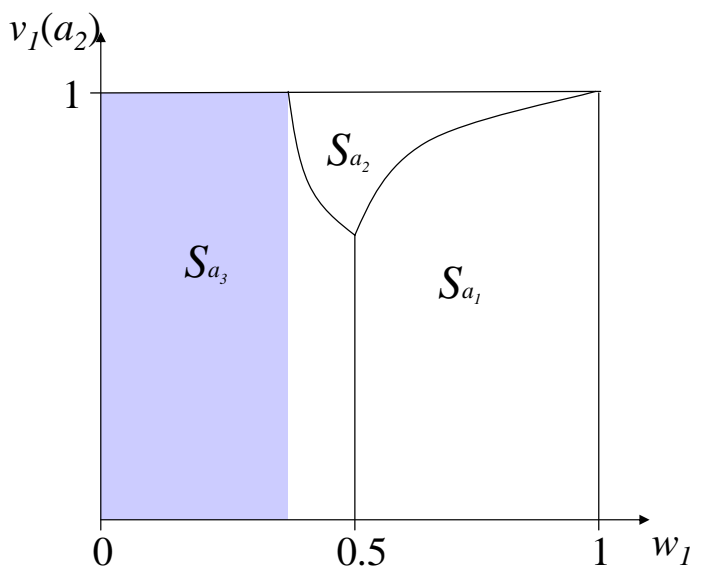

Fig. 2. $S_{a}, a \in A$ for Example 1

Figure 2 illustrates the fact that if we ask "Is $w_{1} \leq 0.41$ ?" and if the answer is "yes," then $a_{3}$ is identified as the most preferred alternative. Note that only partial information about the value of $w_{1}$ and no information about the value of $v_{1}\left(a_{2}\right)$ was required in order to determine the most preferred alternative. As another example, if the question "Is $v_{1}\left(a_{2}\right) \leq 0.7$ ?" elicits a response of "yes," then we can eliminate $a_{2}$ from further consideration. Asking the question "Is $w_{1} \leq 0.5$ ?" would then determine the most preferred alternative.

Questions generate not only responses but also tend to raise the DM's expectations that progress is being made toward problem resolution. However, assume that for some behavioral or organizational reason, questions of the form "Is $v_{1}\left(a_{2}\right) \leq \psi$ ?" cannot be asked. (For example, consider the case where additional information regarding cost attributes values is not immediately available, or if discussion of an attribute is expected to result in extended debate among members of the decision-making body.) If the answer to the question "Is $w_{1} \leq 0.41$ ?" is "no," then we note that problem resolution cannot occur by simply asking questions of the form "Is $w_{1} \leq \beta$ ?". Under these circumstances, there is good reason to stop the facilitation process with the explanation that it is no longer possible to reduce the non-dominated set to a single alternative. This tactic would at least minimize the consternation likely to result from a process of asking questions and receiving responses that are guaranteed to result in no progress toward problem resolution. The intent of the research presented in this paper is to identify when such a situation exists and present a priori conditions that insure it cannot exist.

Situations where problem resolution cannot occur, illustrated in the example above when questions of the form "Is $v_{1}\left(a_{2}\right) \leq \psi$ ?" cannot be asked, have served to motivate our study of resolvability. Consider the defense communications problem described in [5]. As part of the problem resolution process, the primary author facilitated a group decision-making session described as follows. Each of the DMs had used his staff to determine the value scores for one of the attributes. Several of these value scores were imprecise. Staff members were not accessible during the decision-making session. The DMs only felt that they could provide weight information, and hence the session was focused only on weight elicitation and, they expected, problem resolution. To avoid controversy (large dollar amounts were involved and several of the DMs appeared to have personal stakes in the outcome), the facilitator initially elicited weights imprecisely. This effort produced a non-dominated set containing several alternatives. The group wanted to find a single most preferred alternative. More precise weight elicitation produced no change in the non-dominated set. Precise weights were eventually elicited with considerable time, stress, and effort. This elicitation did not remove any of the non-dominated alternatives, the session was considered a failure and problem resolution eventually was achieved through unaided discussion. This experience identified a real-world situation in which problem resolution was not attainable and motivated the resolvability research presented in this paper.

\section{B. Related Work}

As we have discussed, a total ordering of alternatives may not be necessary for problem resolution. A substantial body of literature exists which seeks to address the problem of generating a partial ordering of alternatives under MAUT, which may be sufficient for decision-making. Fishburn [6], Hannan [7], Kirkwood and Sarin [8], Hazen [9], Weber [10], Barron and Barrett [11], Park and Kim [12], and Athanassopoulos and V. V. Podinovski [13] each outline techniques for generating a partial ordering given linear constraints. The proposed methods differ in terms of the types of constraints considered (for example, using ordinal ranking of unknown quantities [7], [12], bounds [6], or pairwise comparisons of alternatives [8]) and in the notions of dominance employed (strict [8], mixed [13], [6], [10], or weak [9]). 
Sarin [14], White et al. [3] and Weber [15] recognized the need to gather additional information from a DM in those cases where the partial ordering of alternatives is insufficient for problem resolution, although the precise nature of the additional information sought is defined generally. Guidance in formulating questions for use in knowledge acquisition is addressed by Rios-Insua and Mateos [16], who present an algorithm that indicates "good" pairwise comparisons questions to ask. However, this algorithm does not distinguish between questions that are "good" and those that might be better (i.e. optimal in some sense). Chajewska, Koller and Parr [17] select utility elicitation questions such that the expected value of information is maximized at each stage. Rao [18] deals directly with a smaller version of this problem, namely determining an optimal sequence of pairwise comparisons over a set of alternatives with two attributes. The present work extends results from Holloway and White [2], which utilizes information theory in support of optimal question selection for alternative selection; Abbas [19] later applied a similar technique in selecting standard gamble-type questions for determination of utility values, given a total ordering of (uncertain) decision outcomes.

We remark that since we assume that a question posed to a group of DMs always elicits a unanimous response, Arrow's Impossibility Theorem (see, for example, [20]) is not applicable. Even so, our results will demonstrate that complete and precise agreement among the DMs to questions asked may still result in an unresolved problem. If our unanimity assumption is removed, then issues addressed by the Theorem may become applicable and represent a topic for future research.

This paper is organized as follows. We formulate the question selection problem as a Markov decision process in Section II. In Section III we state preliminary results from the dynamic programming literature. In Section IV, we examine the issue of resolvability, and present conditions to aid the facilitator in determining if asking additional questions can possibly lead to problem resolution. We also present conditions that guarantee the existence of a question-response policy that will identify a most preferred alternative in a finite number of questions. Additionally, we present conditions that guarantee the existence of a question-response policy that will identify a most preferred alternative as the number of questions asked goes to infinity. These two sets of conditions are easily identified a priori and guarantee that at least questions can be asked that lead toward problem resolvability. Conclusions are presented in the final section.

\section{The QSP PROBlem Formulation}

\section{A. State Space}

Let $N$ be the number of imprecise parameters associated with the QSP, let $\bar{S} \subseteq \Re^{N}$ be the set of all possible parameter values, and let $\mathcal{S}$ be the collection of all subsets of $\bar{S}$. (Technically, we restrict $\mathcal{S}$ to be the Borel sets of $\bar{S}$; however, this technicality is of little consequence in applications.) We consider each element in $\bar{S}$ to be a representation of preference. If $s^{*} \in \bar{S}$ represents the actual, and initially unknown, preference of the DM, then our intent is to know $s^{*}$ sufficiently well enough via set inclusion to select a most preferred alternative. Thus, $\mathcal{S}$ is the state space of the QSP.

EXAMPLE 2. For the simplest ISMAUT model described in Section I, let $\bar{W}=\left\{w \geq 0: \sum_{i \in I} w_{i}=1\right\}$ be the set of trade-off weights, $\overline{V_{i}}=[0,1]^{|A|}$ be the set of value scores associated with attribute $i \in I$, where $|A|$ is the cardinality of set $A$, and $\bar{V}=\times_{i \in I} \overline{V_{i}}$ be the set of all value scores. Then $\bar{S}=\bar{V} \times \bar{W}$.

\section{B. Question Set}

We assume $Q$ is the (not necessarily finite) set of all questions available to the facilitator and that $Q_{t}(S) \subseteq Q$ is the set of questions permitted to be asked at decision epoch $t$ when the current state is $S \in \mathcal{S}$. For each $q \in Q$, let $R(q)$ be the finite set of all responses to question $q$. The set $(q, r) \in \mathcal{S}$ represents the set of all parameter values that are consistent with response $r \in R(q)$ to question $q \in Q$. For example, if $q=$ "Is $w_{i} \leq 0.5$ ?" and $r=$ "Yes," then $(q, r)=\left\{w_{i} \leq 0.5\right\}$. Thus all answers are assumed truthful with respect to $s^{*}$.

EXAMPLE 3. For the simplest ISMAUT model described in Section I, we consider three types of questions. First, let $Q_{p w}$ be the set of all pairwise comparisons. Thus, $Q_{p w}$ is comprised of the collection of questions of the form "Is $a_{i}$ at least as preferred as $a_{j}$ ?" for all $i=1, \ldots,|A|-1$, and $j, j=i+1, \ldots,|A|$, where $|A|$ is the cardinality of the set $A$. Thus $\left|Q_{p w}\right|=|A|(|A|-1) / 2$, and hence $Q_{p w}$ is finite. For $q \in Q_{p w}, R(q)=\{$ Yes, No $\}$. If $q=$ "Is $a_{i}$ at least as preferred as $a_{j}$ ?" and $r=$ "Yes," then $(q, r)=\{(v, w)$ : $\left.w v\left(a_{i}\right) \geq w v\left(a_{j}\right)\right\}$.

Second, let $Q_{w}$ be the (uncountable) set of questions of the form "Is $w_{i} \leq \psi$ ?" where for all $q \in Q_{w}, R(q)=\{$ Yes, No $\}$. If $q=$ "Is $w_{i} \leq \psi$ ?" and $r=$ "Yes," then $(q, r)=\{(v, w)$ : $\left.w_{i} \leq \psi\right\}$.

Third, let $Q_{v}$ be the (uncountable) set of questions of the form, "Is $v_{i}(a) \leq \beta$ ?" where for all $q \in Q_{v}, R(q)=\{$ Yes, No $\}$. If $q=$ 'Is $v_{i}(a) \leq \beta$ ?" and $r=$ "Yes," then $(q, r)=$ $\left\{(v, w): v_{i}(a) \leq \beta\right\}$.

\section{Probability Measure}

Let $P$ be a given probability measure on $\mathcal{S}$. We say that a finite collection of subsets of $\bar{S},\left\{S_{k}, k=1, \ldots, K\right\}$, is a (finite) partition of $\bar{S}$ if and only if $\cup_{k} S_{k}=\bar{S}, P\left(S_{k}\right) \neq 0$ for all $k \in\{1, \ldots, K\}$, and $P\left(S_{k} \cap S_{k^{\prime}}\right)=0$ if $k \neq k^{\prime}$. We assume that for all $q \in Q,\{(q, r), r \in R(q)\}$ is a partition of $\bar{S}$.

Conforming to the notation in [2], define $\tilde{\sigma}(r, S, q)=P[S \cap$ $(q, r)] / P(S)$ for all $S$ such that $P(S)>0, q \in Q$, and $r \in$ $R(q)$. It is easily shown that $\sum_{r \in R(q)} \tilde{\sigma}(r, S, q)=1$ for all $q \in Q$ and $S \in \mathcal{S}$ such that $P(S)>0$.

We provide the following interpretation of these terms and use this interpretation throughout the remainder of the paper. Let $P(S)$ be the probability that $s^{*}$ is a member of $S$. The term $\tilde{\sigma}(r, S, q)$ is the probability that $r \in R(q)$ will be the response to question $q \in Q$, assuming $s^{*} \in S$. 


\section{Dynamics of the Parameter Value Set}

Let $S_{t} \in \mathcal{S}$ be the parameter value set just before the $t^{\text {th }}$ question is asked, let $q_{t}$ be the question asked at epoch $t$, and let $r_{t}$ be the response. Then $S_{t+1}=S_{t} \cap\left(q_{t}, r_{t}\right) \in \mathcal{S}$ with probability $\tilde{\sigma}\left(r_{t}, S_{t}, q_{t}\right)$. Note that $S_{t+1} \subseteq S_{t}$, for all $t$, indicating a learning feature of the model.

\section{E. Decision Rules and Policies}

We assume that the selection of $q_{t}$ is based on knowledge of $S_{t}$ and $t$. Let $T<\infty$ be the maximum number of questions that can be posed. A decision rule is a function $d: \mathcal{S} \rightarrow Q$, and a (question-response) policy is a sequence of decision rules, $\pi=\left\{d_{1}, \ldots, d_{T}\right\}$, where $d_{t}$ is the decision rule used to select the $t^{t h}$ question, and hence $d_{t}\left(S_{t}\right)=q_{t} \in Q_{t}\left(S_{t}\right)$.

\section{F. Cost Structure, Criterion, and Problem Objective}

We assume $c(S, q)$ is the cost of asking question $q \in Q$, given parameter value set $S \in \mathcal{S}$. Assume $\bar{c}(S)$ is the terminal cost associated with parameter value set $S \in \mathcal{S}$ after all questions have been asked.

A policy generates an expected cost $C^{\pi}(S)$, the criterion, where

$$
C^{\pi}(S)=E_{S}^{\pi}\left\{\sum_{t=1}^{T} c\left(S_{t}, q_{t}\right)+\bar{c}\left(S_{T+1}\right)\right\}
$$

and where $E_{S}^{\pi}$ is the expectation operator, conditioned on the use of policy $\pi$ and that $S_{1}=S$. The problem objective is to determine a policy $\pi^{*}$, called an optimal policy, such that $C^{\pi^{*}}(S) \leq C^{\pi}(S)$ for all $\pi$ and $S$.

An optimal policy represents advice to the facilitator as to which question to ask next. More specifically, let $\pi^{*}=$ $\left\{d_{1}^{*}, \ldots, d_{T}^{*}\right\}$. Then, if $S_{t}=S, q_{t}$ should be selected to be $d_{t}^{*}(S)$.

Of course, $d_{t}^{*}\left(S_{t}\right)$ represents a good choice for $q_{t}$ only if the model accurately depicts the objectives of the alternative selection process. We remark that the notion of an alternative is not explicit in the above problem formulation. We now present an example showing how the alternatives and the objective of the alternative selection process provide a basis for the descriptions of the cost structure $(c, \bar{c})$.

EXAMPLE 4. For the simplest ISMAUT model described in Section I, assume we wish to find a most preferred alternative in $A$. Let $S_{a}=\left\{(v, w): w v(a) \geq w v\left(a^{\prime}\right)\right.$ for all $\left.a^{\prime} \in A\right\}$, and note that $\left\{S_{a}, a \in A\right\}$ is a partition of $\bar{S}$. Let $\bar{c}(S)=0$ if $S \subseteq S_{a}$ for some $a \in A$ and $\bar{c}(S)=1$ otherwise. If $c(S, q)=0$, then an optimal policy will select questions so as to maximize the probability of problem resolution. Assume $c(S, q)=0$ if $S \subseteq S_{a}$ for some $a$, and $c(S, q)=1$ otherwise. Then an optimal policy will also attempt to minimize the number of questions required for problem resolution.

\section{A Dynamic Programming Approach to QUESTION SELECTION}

We now apply dynamic programming to determine $\pi^{*}$, $C^{\pi^{*}}$, and the concomitant optimality equation. The following well-known result presents an optimality equation and its implications for the QSP. A proof may be found in [21, page 84].

Theorem 1: Let

$$
\begin{aligned}
\tilde{c}_{t}(S)= & \inf _{q}\left\{c(S, q)+\sum_{r} \tilde{\sigma}(r, S, q) \tilde{c}_{t+1}[S \cap(q, r)]\right\}, \\
& t=1, \ldots, T, \\
\tilde{c}_{T+1}(S)= & \bar{c}(S) .
\end{aligned}
$$

Then $\tilde{c}_{1}(S)=\inf _{\pi} C^{\pi}(S)$. Further, a policy constructed from decision rules that cause the above infimum to be attained for all $t$ is necessarily and sufficiently an optimal policy.

The uncountably infinite cardinality of the state space insures that the QSP in general is intractable. A two-step procedure and several key assumptions that guarantee $\tilde{c}_{t}$, for all $t$, has a finite representation and hence the QSP is potentially computable is presented in [2] for the case where value scores are precise. These results are easily extended to the more general case considered here.

\section{ResolvabiLity}

Example 1 presents a situation where there exists no policy that can lead to the identification of a most preferred alternative if questions are restricted to be of the form "Is $w_{1} \leq \beta$ ?" and if $S_{1} \subseteq\left\{(v, w): w_{1} \geq 0.41\right\}$. As indicated earlier, knowing when such a situation exists could be of significant value to a facilitator. We now determine the probability that such a situation may exist. We also give conditions guaranteeing the existence of a policy that will lead to problem resolution with probability one.

Let $\mathcal{S}^{\prime} \subseteq \mathcal{S}$ be the collection of sets such that $S \in \mathcal{S}^{\prime}$ if and only if there exists an $a \in A$ such that $S \subseteq S_{a}$. Then $P\left(S_{t} \in\right.$ $\left.\mathcal{S}^{\prime}\right)$ is the probability that the problem is resolved before the $t^{t h}$ question is asked and hence represents the probability of interest.

We remark that once a QSP is resolved, it remains resolved. This is due to the fact that $S_{t+1} \subseteq S_{t}$ for all $t$ and that if $S \in \mathcal{S}^{\prime}$ and $S^{\prime} \subseteq S$, then $S^{\prime} \in \mathcal{S}^{\prime}$. Thus, $P\left(S_{t} \in \mathcal{S}^{\prime}\right) \leq$ $P\left(S_{t+1} \in \mathcal{S}^{\prime}\right)$.

Observe that if there is a policy such that $P\left(S_{t} \in \mathcal{S}^{\prime}\right)=1$ for some $t$ or perhaps in the limit as $t \rightarrow \infty$, then the facilitator can be assured that the situation illustrated in Example 1 when questions are restricted to the form "Is $w_{1} \leq \beta$ ?" cannot happen. However, if $P\left(S_{t} \in \mathcal{S}^{\prime}\right)$ is close to or equal to zero, then there is good reason to consider stopping the QSP with the problem unresolved.

We begin this section by applying Theorem 1 to compute $P\left(S_{t} \in \mathcal{S}^{\prime}\right)$ and determine a policy that will maximize it. We then present conditions that guarantee $P\left(S_{t} \in \mathcal{S}^{\prime}\right)$ converges to 1 as $t \rightarrow \infty$ and, better yet, conditions that guarantee $P\left(S_{t} \in \mathcal{S}^{\prime}\right)=1$ for finite $t$.

\section{A. Determination of $P\left(S_{T+1} \in \mathcal{S}^{\prime}\right)$}

We now present simple iterative procedures for determining:

(i) $\quad P\left(S_{t} \in \mathcal{S}^{\prime}\right)$, given $\pi$.

(ii) $\sup _{\pi} P\left(S_{t} \in \mathcal{S}^{\prime}\right)$ and a policy that causes the supremum to be attained, if such a policy exists. 


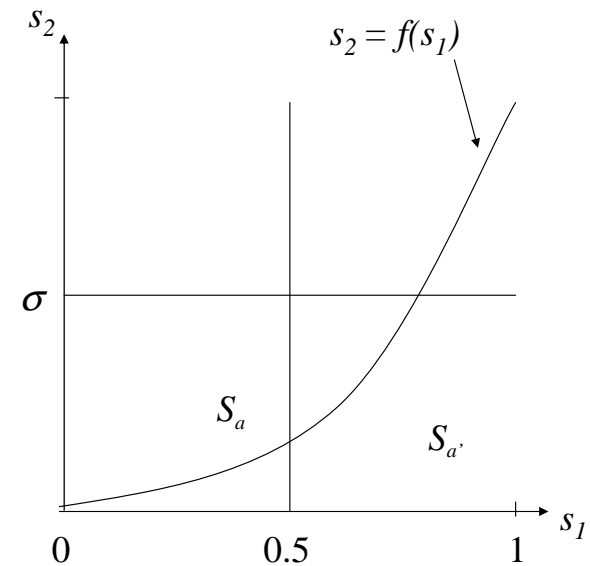

Fig. 3. The Partition $\left\{S_{a}, S_{a^{\prime}}\right\}$ for $f\left(s_{1}\right)=s_{1}^{2}$.

Such information can help the facilitator guide the questionresponse process, in the case of a given policy, and can provide guidance to the facilitator regarding whether or not to stop the process, in the case of the probabilities. Proofs of the following corollaries to Theorem 1 follow directly from the proof of Theorem 1.

Corollary 1: Let $\bar{c}_{T+1}(S)=1(=0)$ if $S \notin \mathcal{S}^{\prime}\left(S \in \mathcal{S}^{\prime}\right)$ and $\pi=\left(d_{1}, \ldots, d_{T}\right)$. Then $P\left(S_{T+1} \in \mathcal{S}^{\prime}\right)$, given $\pi$, equals $1-c_{1}^{\pi}\left(S_{1}\right)$ where for $q_{t}=d_{t}\left(S_{t}\right), t=1, \ldots, T$,

$$
c_{t}^{\pi}\left(S_{t}\right)=\sum_{r} \tilde{\sigma}\left(r, S_{t}, q_{t}\right) c_{t+1}^{\pi}\left[S_{t} \cap\left(q_{t}, r_{t}\right)\right]
$$

and $c_{T+1}^{\pi}\left(S_{T+1}\right)=\bar{c}_{T+1}\left(S_{T+1}\right)$.

Corollary 2: Let $\bar{c}_{T+1}(S)=1(=0)$ if $S \notin \mathcal{S}^{\prime}\left(S \in \mathcal{S}^{\prime}\right)$ and $c(S, q)=0$ for all $S$ and $q$. Then,

$$
\sup _{\pi} P\left(S_{T+1} \in \mathcal{S}^{\prime}\right)=1-c_{1}^{*}\left(S_{1}\right)
$$

where $c_{1}^{*}$ is given in Theorem 1. Further, a policy constructed from decision rules that cause the infimum in Equation 2 to be attained for all $t$ is necessarily and sufficiently an optimal policy.

Example 5: We now illustrate the use of Corollary 2. Let $\mathrm{N}=2$, $\bar{S}=\left\{0 \leq s_{n} \leq 1, n=1,2\right\}, S_{1}=\bar{S}$, and $T=3$, and assume the solution partition $\left\{S_{a}, S_{a^{\prime}}\right\}$ is given as described in Figure 3 , where $s_{2}=f\left(s_{1}\right)=\left(s_{1}\right)^{2}$ represents the boundary between $S_{a}$ and $S_{a^{\prime}}$. Let $d_{1}(S)=$ "Is $s_{1} \leq 0.5$ ?" and $d_{2}(S)=$ "Is $s_{2} \leq \sigma$ ?" for all $0 \leq \sigma \leq 1$. Assume $P(S)$ is the area of $S$; i.e. assume $P$ is a uniform distribution on $\bar{S}$. It is then straightforward to show that

$$
\begin{aligned}
P\left(S_{3} \in \mathcal{S}^{\prime}\right) & =(1-\sigma) / 2 & & \sigma>1 / 4 \\
& =1 / 2 & & \sigma=1 / 4 \\
& =\sigma / 2 & & \sigma<1 / 4,
\end{aligned}
$$

which is depicted graphically in Figure 4.

Thus, an optimal policy is "Is $s_{1} \leq 0.5$ ?" and "Is $s_{2} \leq$ $f(0.5)$ ?" and the largest probability for problem resolution after two questions is 0.5 . We remark that if either questions of the form "Is $s_{1} \leq \alpha$ ?" or "Is $s_{2} \leq \beta$ ?" were allowed,

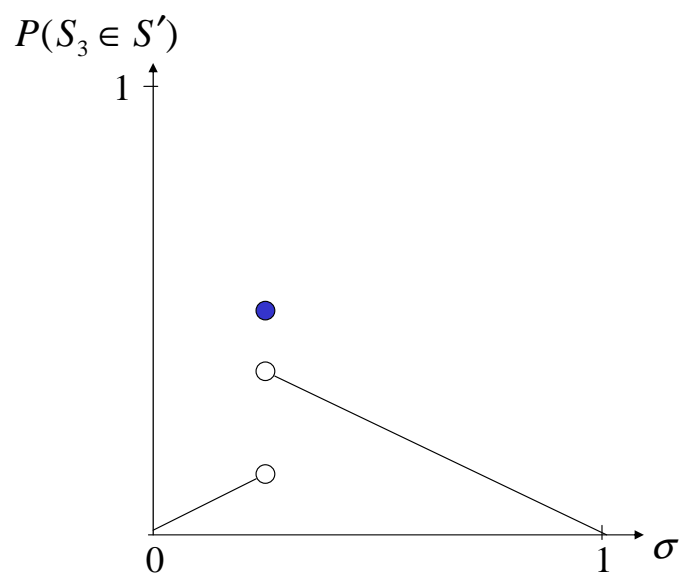

Fig. 4. $P\left(S_{3} \in \mathcal{S}^{\prime}\right)$, as a function of $\sigma$.

but not both, then the QSP would be unresolvable and hence $P\left(S_{t} \in \mathcal{S}^{\prime}\right)=0$ for all $t$

\section{B. Conditions for Finite Resolvability}

We now present conditions guaranteeing that the QSP is resolvable in a finite number of questions.

Definition 1: The question selection problem is said to be finitely resolvable if $\exists T<\infty$ and $\pi$ such that $P\left(S_{T} \in \mathcal{S}^{\prime}\right)=1$.

Definition 2: For partitions $X=\left\{X_{\beta}, \beta \in \mathcal{B}\right\}$ and $Y=$ $\left\{Y_{\sigma}, \sigma \in \Sigma\right\}$, define $X \cap Y$ as the collection of all possible sets $X_{\beta} \cap Y_{\sigma}$ for some $\beta \in \mathcal{B}$ and $\sigma \in \Sigma$.

Definition 3: For partitions $X=\left\{X_{\beta}, \beta \in \mathcal{B}\right\}$ and $Y=$ $\left\{Y_{\sigma}, \sigma \in \Sigma\right\}$, we say $X$ is at least as fine as $Y$ if and only if for every $\sigma \in \Sigma$ there is a $\beta \in \mathcal{B}$ such that $X_{\beta} \subseteq Y_{\sigma}$.

It is easy to show that if $X$ and $Y$ are partitions, then so is $X \cap Y$. Further, $X \cap Y$ is at least as fine as either $X$ or $Y$.

Definition 4: Let $P^{Q}=\cap_{q \in Q}\{(q, r), r \in R(q)\}$ be the partition generated by the question set $Q$.

Theorem 2: Assume $\exists Q^{\prime} \subseteq Q,\left|Q^{\prime}\right| \leq T<\infty$, such that $P^{Q^{\prime}}$ is at least as fine a partition as $\left\{S_{a}, a \in A\right\}$. Then the problem is finitely resolvable in $T$ questions.

Proof: Construct $\pi=\left\{d_{1}, \ldots, d_{T}\right\}$ as follows. For $t \leq T$, let $d_{t}(S)=q_{t} \in Q^{\prime}$ for all $S \subseteq \bar{S}$, where for $t, \tau \leq\left|Q^{\prime}\right|, q_{t} \neq$ $q_{\tau}$ if $t \neq \tau$. Then for any $S_{1} \subseteq \bar{S}, S_{\left|Q^{\prime}\right|+1}=S_{1} \cap_{t=1}^{\left|Q^{\prime}\right|}\left(q_{t}, r_{t}\right)$. By assumption, there exists an $a \in A$ such that $S_{\left|Q^{\prime}\right|+1} \subseteq S_{a}$.

We now present a simple, practical sufficient condition for finite resolvability, a corollary to Theorem 2 .

Corollary 3: If $Q_{p w} \subseteq Q$, then the problem is finitely resolvable for any $S_{1}$ when $T \geq|A|-1$.

Proof: Consider the following policy. Let $d_{t}\left(S_{t}\right)=\left(a_{i}, a_{j}\right)$, where $i<j$, and $a_{i}, a_{j} \in N D\left(S_{t}\right)$ are such that $\nexists a_{m} \in$ $N D\left(S_{t}\right)$ such that $m<i$ or $i<m<j$. After receiving a response, determine $N D\left(S_{t+1}\right)$. If $\left|N D\left(S_{t+1}\right)\right|=1$, stop. Otherwise, continue asking questions. Because the questions asked are all comparisons within the non-dominated set, every response indicates a new dominance relationship within that 
set, and hence reduces the size of the set by 1 each time. Under this policy, $\left|N D\left(S_{t+1}\right)\right|=\left|N D\left(S_{t}\right)\right|-1$. Since $\left|N D\left(S_{1}\right)\right| \leq$ $|A|,\left|N D\left(S_{|A|-1}\right)\right|=1$ under this policy.

Thus, if all pairwise comparisons are permissible, then the problem is guaranteed to be finitely resolvable.

We remark that $Q_{p w} \subseteq Q$ is a sufficient condition of finite resolvability but is not necessary. Consider Example 1, and let $Q$ be comprised of the following questions: Is $v_{1}\left(a_{2}\right) \leq 0.7$ and $w_{1} \leq 0.5$ ? Is $v_{1}\left(a_{2}\right) \leq 0.7$ and $w_{1} \geq 0.5$ ? Is $a_{2}$ at least as preferred as $a_{1}$ ? Is $a_{2}$ at least as preferred as $a_{3}$ ? We note that $Q$ does not contain all questions in $Q_{p w}$ yet does satisfy the conditions in Theorem 2 .

It might be claimed that permitting the use of pairwise comparisons eliminates the need for eliciting information about the weights and attribute values. In reality, the use of pairwise comparisons is always available; however, asking a pairwise comparison question may not promise problem resolution. It has been our experience that pairwise comparisons of alternatives in the non-dominated set can produce discord in a group alternative selection session if members of the group champion alternatives. In such situations, we have found it useful to ask pairwise comparison questions only involving alternatives that have been eliminated from the non-dominated set. Such alternatives are unlikely to retain committed champions, and the concomitant pairwise comparisons can be quite informative for reducing $S$ and $N D(S)$.

\section{Resolvability in the Limit}

We now present assumptions that guarantee the QSP is resolvable if the facilitator is not limited to a finite number of questions. In reality, the number of questions that a facilitator can ask is small. However, resolvability in the limit implies at least the potential for problem resolution and hence that asking good questions can reduce the non-dominated set of alternatives.

Definition 5: The problem is resolvable in the limit if for any $\epsilon>0$, there is a policy $\pi$ and a time $T<\infty$ such that $P\left(S_{t} \in \mathcal{S}^{\prime}\right) \geq 1-\epsilon$ for all $t \geq T$.

Equivalently, the question selection problem is resolvable in the limit if there is a policy $\pi$ such that $\lim _{t \rightarrow \infty} P\left(S_{t} \in \mathcal{S}^{\prime}\right)=1$.

The following theorem presents conditions that guarantee resolvability in the limit.

Theorem 3: Assume there is a policy $\pi$, a scalar $\delta$, where $0<\delta \leq 1$, an integer $M<\infty$ and an integer $T<\infty$, such that for all $t \geq T, \mathrm{P}\left(S_{t+M} \in \mathcal{S}^{\prime} \mid S_{t} \notin \mathcal{S}^{\prime}\right) \geq \delta$. Then the QSP is resolvable in the limit.

Proof: Assume without loss of generality that $S_{1} \notin \mathcal{S}^{\prime}$. Note that $P\left(S_{t+M} \in \mathcal{S}^{\prime}\right)=P\left(S_{t} \in \mathcal{S}^{\prime}\right)+P\left(S_{t+M} \in \mathcal{S}^{\prime} \mid S_{t} \notin\right.$ $\left.\mathcal{S}^{\prime}\right)\left[1-P\left(S_{t} \in \mathcal{S}^{\prime}\right)\right]$. It then follows that for $t \geq T$,

$$
1-P\left(S_{t+M} \in \mathcal{S}^{\prime}\right) \leq(1-\delta)\left[1-P\left(S_{t} \in \mathcal{S}^{\prime}\right)\right]
$$

where $0 \leq(1-\delta)<1$. Thus, $\lim _{t \rightarrow \infty} P\left(S_{t} \in \mathcal{S}^{\prime \prime}\right)=1$.

The proof of the following corollary determines $\delta$ and $M$ and presents an approach for constructing a policy $\pi$, as required in Theorem 3. Let $Q_{S}$ be the set of all questions of the form "Is $s_{n} \leq \alpha$ ?" for all $n=1, \ldots, N$ and all $\alpha$.

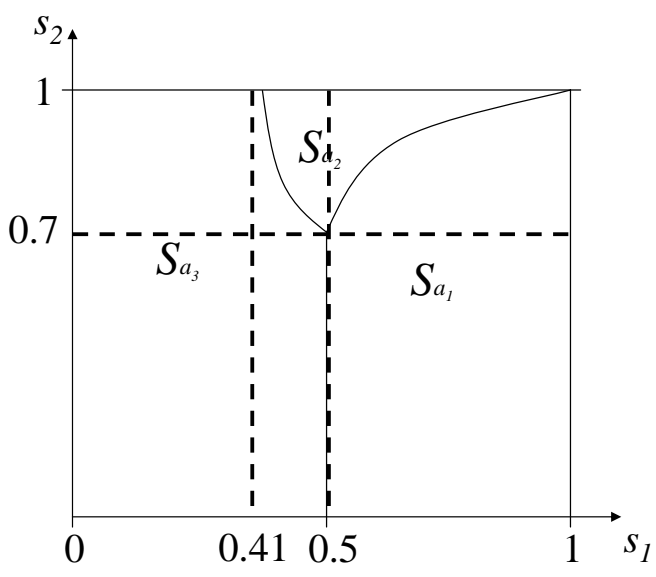

Fig. 5. $\mathrm{T}=3$ Partition of $S_{1}$

Corollary 4: Let $\bar{S}$ be the unit cube in $\Re^{N}$ and let $P(S)$, $S \in \mathcal{S}$ be the volume of $S$. Assume:

(i) there exists a $T<\infty$ such that $S_{T} \subseteq S_{a} \cup S_{a^{\prime}}, a, a^{\prime} \in$ $A, a \neq a^{\prime}$;

(ii) $S_{T}=\{s: l \leq s \leq u\}$, where $\mathbf{l}=\left(l_{1}, \ldots, l_{N}\right)$ and $\mathbf{u}=\left(u_{1}, \ldots, u_{N}\right)$;

(iii) the boundary between $S_{a}$ and $S_{a^{\prime}}$ is described by $s_{N}=f\left(s_{1}, \ldots, s_{N-1}\right)$ where $s=\left(s_{1}, \ldots, s_{N}\right)$ and where $f$ is monotone; and

(iv) $Q_{S} \subseteq Q$.

Then the QSP is resolvable in the limit.

Proof: Let $\bar{s}_{n}=\left(u_{n}+l_{n}\right) / 2$ for $n=1, \ldots, N-1, \bar{s}_{N}=$ $f\left(\bar{s}_{1}, \ldots, \bar{s}_{N-1}\right)$ and $\overline{\mathbf{s}}=\left(\bar{s}_{1}, \ldots, \bar{s}_{N}\right)$. By assumption, $\overline{\mathbf{s}} \in$ $S_{t}$. Without loss of generality, assume $s \leq s^{\prime}$ implies $f(s) \leq$ $f\left(s^{\prime}\right)$ and that $\left\{s \in S_{t}: s_{N} \leq f\left(s_{1}, \ldots, s_{N-1}\right)\right\} \subseteq S_{a}$ and $\left\{s \in S_{t}: s \geq f\left(s_{1}, \ldots, s_{N-1}\right)\right\} \subseteq S_{a^{\prime}}$. Let $M=N$, and let $\pi$ be such that it asks the following $N$ questions: "Is $s_{n} \leq \bar{s}_{n}$ ?", $n=1, \ldots, N$. If the response to these questions is "yes," $n=1, \ldots, N-1$, and "no," $n=N$, then $S_{t+N}=\left\{s: l_{n} \leq\right.$ $s_{n} \leq\left(u_{n}+l_{n}\right) / 2, n=1, \ldots, N-1$, and $\left.\bar{s}_{N} \leq s_{N} \leq u_{N}\right\}$ and has volume $V_{1}=\left(u_{N}-\bar{s}_{N}\right) \prod_{n=1}^{N-1}\left(u_{n}-l_{n}\right) / 2$. Since $S_{t+N} \subseteq S_{a^{\prime}}$, the problem is resolved.

Similarly, if the question responses are "no," $n=$ $1, \ldots, N-1$, and "yes," $n=N$, then $S_{t+N}$ has volume $V_{2}=\left(\bar{s}_{N}-l_{N}\right) \prod_{n=1}^{N-1}\left(u_{n}-l_{n}\right) / 2$ and the problem is resolved since $S_{t+N} \subseteq S_{a}$.

We note that the volume of $S_{t}$ is $V=\prod_{n=1}^{N}\left(u_{n}-l_{n}\right)$. Thus $P\left(S_{t+N} \in \mathcal{S}^{\prime} \mid S_{t} \notin \mathcal{S}^{\prime}\right) \geq\left(V_{1}+V_{2}\right) / V=1 / 2^{N-1}$, and hence we can set $\delta=1 / 2^{N-1}$. The proof then follows from Theorem 3.

We remark that assumptions (i)-(iii) in Corollary 4 are quite robust. For illustration, consider Figure 5 (associated with Example 1 and utilizing a more general notation). If we ask the questions "Is $s_{1} \leq 0.41$ ?", "Is $s_{1} \leq 0.5$ ?", and "Is $s_{2} \leq 0.7$ ", then we are guaranteed that assumptions (i)-(iii) of Corollary 4 are valid for all $S_{t} \notin \mathcal{S}^{\prime}, t \geq 3$.

Furthermore, we remark that there may be no upper bound on the number of questions needed for resolvability, given 
the strategy constructed in the proof of Corollary 4, thus justifying the need for resolvability in the limit as a concept. For example, following the proof of Corollary 4, assume $s_{n}^{*}=u_{n}-\epsilon, n=1, \ldots, N$ for given $\epsilon>0$ and that $s^{*} \in S_{a}$. Let the integer $l$ be such that $\epsilon \leq 1 /(l-1)$ and $\epsilon>1 / l$. Then, the QSP will only be resolved after $l$ sets of questions of the form, " Is $s_{n} \leq \bar{s}_{n}$ ?", $n=1, \ldots, N$. Since $\epsilon>0$ may be arbitrarily small, $l$ can become arbitrarily large.

\section{Conclusions}

We presented an example in Section I that indicated that there might not exist a question-response policy for the QSP that can lead to the identification of a most preferred alternative. Based on a Markov decision process model of the QSP, we then presented an approach for determining the probability that the QSP will be unresolved. Additionally, we presented conditions that guarantee the existence of a questionresponse policy that will identify a most preferred alternative in a finite number of questions and conditions that guarantee the existence of a question-response policy that will identify a most preferred alternative as the number of questions asked goes to infinity. Both sets of conditions are easily identified a priori and pertain to the type of question the facilitator is permitted to ask.

In the context of the simple ISMAUT model presented in the Introduction, if a facilitator can ask any pairwise comparison question or ask about the value of any trade-off weight or value score, then problem resolution can be achieved. However, if some or all of the pairwise comparison questions are not permissible and if assessment of some or all of the tradeoff weights and value scores are not allowed, then it may be possible that the QSP will be unresolved.

Thus, in complement with results presented by Holloway and White [2], we have presented results that represent a partial basis for a facilitator support system. Such a system would aid the facilitator in question selection and inform (alert and warn) the facilitator of the probability of resolvability. This latter function presumably would allow the facilitator to better manage the time, effort, and expectations of the DM and better determine when to stop the alternative selection process.

\section{REFERENCES}

[1] R. Keeney and H. Raiffa, Decisions with Multiple Objectives: Preferences and Value Tradeoffs. New York: Wiley, 1976.

[2] H. A. Holloway and C. C. White III, "Question selection for multiattribute decision-aiding," European Journal of Operational Research, vol. 148, no. 3, pp. 525-533, 2003.

[3] C. C. White III, A. P. Sage, and S. Dozono, "A model of multiattribute decision-making and trade-off weight determination under uncertainty," IEEE Transactions on Systems, Man, and Cybernetics, vol. 14, no. 2, pp. 223-229, 1984.

[4] J. F. Bard, "A comparison of the Analytic Hierarchy Process with multiattribute utility theory - a case-study," IIE Transactions, vol. 24, no. 5, pp. 111-121, 1992.

[5] B. S. Stewart, W. T. Scherer, E. A. Sykes, and C. C. White III, "Defense communications decision support using ISMAUT and extensions," in Advanced Technology for $C^{2}$ Systems Engineering (S. Andriole, ed.), AFCEA International Press, 1990.

[6] P. C. Fishburn, "Analysis of decisions with incomplete knowledge of probabilities," Operations Research, vol. 13, no. 2, pp. 217-237, 1965.
[7] E. L. Hannan, "Obtaining nondominated priority vectors for multiple objective decisionmaking problems with different combinations of cardinal and ordinal information," IEEE Transactions on Systems, Man, and Cybernetics, vol. 11, no. 8, pp. 538-543, 1981.

[8] C. W. Kirkwood and R. K. Sarin, "Ranking with partial information: A method and an application," Operations Research, vol. 33, no. 1, pp. $38-48,1983$.

[9] G. B. Hazen, "Partial information, dominance, and potential optimality in multiattribute utility theory," Operations Research, vol. 34, no. 2 , pp. 296-310, 1986.

[10] M. Weber, "Decision making with incomplete information," European Journal of Operational Research, vol. 28, pp. 44-57, 1987.

[11] F. Barron and B. E. Barrett, "Decision quality using ranked attribute weights," Management Science, vol. 42, no. 11, pp. 1515-1523, 1996.

[12] K. S. Park and S. H. Kim, "Tools for interactive multiattribute decisionmaking with incompletely identified information," European Journal of Operational Research, vol. 98, pp. 111-123, 1997.

[13] A. D. Athanassopoulos and V. V. Podinovski, "Dominance and potential optimality in multiple criteria decision analysis with imprecise information," Journal of the Operational Research Society, vol. 48, pp. 142-150, 1997.

[14] R. K. Sarin, "Screening of multiattribute alternatives," OMEGA, vol. 5, no. 4, pp. 481-489, 1977.

[15] M. Weber, "A method of multiattribute decision making with incomplete information," Management Science, vol. 31, no. 11, pp. 1365-1371, 1985.

[16] S. Rios-Insua and A. Mateos, "The utility efficient set and its interactive reduction," European Journal of Operational Research, vol. 105, pp. 581-593, 1998.

[17] U. Chajewska, D. Koller, and R. Parr, "Making rational decisions using adaptive utility elicitation," in Proceedings of the Seventeenth National Conference on Artificial Intelligence, (Austin, Texas), pp. 363-369, American Association for Artificial Intelligence, August 2000.

[18] H. R. Rao, "A choice set sensitive analysis of preference information acquisition about discrete resources," IEEE Transactions on Systems, Man, and Cybernetics, vol. 23, no. 4, pp. 1062-1071, 1993.

[19] A. E. Abbas, "Entropy methods for adaptive utility elicitation," IEEE Transactions on Systems, Man, and Cybernetics A, vol. 34, no. 2, pp. 169-178, 2004.

[20] J. Geanakaplos, "Three brief proofs of Arrow's Impossibility Theorem," Tech. Rep. 1123R3, Cowles Foundation for Research in Economics, Yale University, 2001.

[21] M. Puterman, Markov Decision Processes. Wiley, 1994. 